\title{
Relevance Study of Translation Process
}

\author{
Bowang Chen ${ }^{1, ~ a, ~ M i n h u a ~ D o n g ~}{ }^{2, b}$ \\ ${ }^{1}$ Nanchang Institute of Science and Technology, Nanchang, Jiangxi, 330108 \\ ${ }^{2}$ Jiangxi Normal University, Nanchang, Jiangxi, 330027 \\ a email:994535324@qq.com, bemail:nick20031001@sohu.com
}

Keywords: Translation Process, Relative Characteristics, Relevance Study

\begin{abstract}
With the development of society, more and more cultural exchanges between East and West have taken place. More and more excellent cultures have gone abroad and become the spiritual food for the people of all countries and the prosperity of the diversity of world cultures. In the process of cultural communication, translation is the main part of cultural transmission. However, due to the different theories of translation, the translation methods, translation forms and translation effects under the guidance of theory are different, resulting in different translation practices. The article starts with the analysis of parameters, transcoding and isomorphism, explores the aspects of technology and cognition, and conducts research and analysis on the relevance between categories of translation theories and translation practice.
\end{abstract}

\section{Introduction}

With the deepening of the opening up to the outside world, the international exchanges have become increasingly close. Translation is an important means to bridge the communication. In the process of translation, the translatability of language and the essence of language are also very different. In the process of translation work, only the positive correlation between translation theory and translation Research and analysis, to enhance the use of research results, can effectively improve the quality of translation, in order to better promote the translation work. In the process of human development, language occupies an important position, at the same time, it is the carrier of human language and communication. Whichever way of language communication basically has the sameness and is also called isomorphism. The so-called language isomorphism mainly refers to the same structure, but also the essence of expression in different languages. The isomorphism existing between different languages is the basis for the two languages to be transla- ted. Moreover, during the process of translation, the two languages only have isomorphism to carry out corresponding translation work. The isomorphism mainly refers to the inter-language Commonality. In the course of the continuous development of society, human languages are relatively independent in the study of diaspora status, and there is a unique way of expression among different languages. The reason for such situation is mainly due to the isomorphism of language Although the languages are different, the mutual understanding of various languages can still be achieved through transcoding. The so-called language transcoding mainly refers to the different languages through mutual transformation, can understand each other, in the actual implementation of transcoding work, language information, cultural background and other content can be displayed through the language structure.

\section{Translation Theory Classification}

Technical level translation theory. The translation theory, which is interpreted from the technical level, has a strong guiding significance both in operation and in practice. The commonly-used theory of Cinda gives guidance to the conversion of specific parameters in translation practice. The more famous Tytler translation concept abroad is a typical theory of technical translation. Newmark's translation theory lies between the technical level and the epistemological level: on the 
one hand, it discusses the specific principles of translation; on the other hand, it studies the principle, nature of translation and its relationship with other disciplines. Nida has high attainments and achievements in both translation theory and practice, and in the process of discussing the relationship between translation theory and practice, Nida has not completely translated translation into epistemological level. In his famous two articles, "The Theory and Practice of Translation" and "The Nature of Translating," he believes that translation should be reader-centered, focusing on the dynamic equivalence of translation functions and spending a lot of ink on the article Translation of practical issues. The theory discussed by Nida is also limited to the translatability between languages, the evaluation methods and standards of translation achievements.

Understanding level translation theory. At the cognitive level, the translation theory focuses on the translator's perspective on the nature of the language, that is, the problem of translatability between different languages. At the epistemological level, translation theories emphasize the universality of languages, the isomorphism of languages and the translatability of languages from a macroscopic perspective under the condition of being isomorphic. Lefevere and Basnett are the representatives of the theory of epistemological translation. They study the importance of ideology in the process of translation and emphasize the importance of translators' values in translation. They think that ideology is composed of conceptual system, which covers the concepts, attitudes, opinions and opinions of specific groups in special historical period in their contents. Ideology directly affects the translator's understanding and excavation of the text. Both of them think that translators The reason why the translation process can play an important role is that it takes on the responsibility of rewriting.

Translation Theory at Non - Translation Level. What the author should emphasize here is that those seemingly discussing translation theories may not belong to the category of translation theory in essence. For example, Derrida and Quinn's problem of hermeneutic discussion do not belong to the problem of translation practice between languages; Habermas's structural theory is not in essence a category of translation theory. In the same way, Heidegger's translation of "using the existence of the world instead of the soul" is not in the category of translation theory because it deals with philosophical issues. He believes that it is difficult to translate the native language The meaning of deconstruction contained in the philosophical existence is completely transformed into another language.

\section{The relationship between translation theory and translation practice}

The Relationship between Translation Theory and Translation Practice. Translation theory comes from translation practice, which is explored and refined in long-term translation practice. Its main function is to test the effect of translation works and translation activities. If we overemphasize the guiding role of translation theory in translation practice, we can only put the cart before the horse so that we can overwhelm the truth. Our translationist Zhang Meifang also thinks that translation theory is the interpretation and prediction of translation works. At the same time, we also need to recognize the evaluation and appreciation role of translation theory on translation works, and evaluate the advantages and disadvantages of translation versions in translation theory In the process, we can elaborate and analyze the deep reasons of its advantages and disadvantages so as to further promote the progress and development of translation practice.

Nowadays, translation has broken through the category of operational theory at the technical level. The translation process is essentially a cultural communication process involving a wide range of levels and complexities. The reading, comprehension and interpretation of the text itself is extremely complicated, and the translation activity needs to express the essence and essence of the text across languages and cultures. The difficulty can be imagined. In the process of thinking about the relationship between translation theory and translation practice, we can not simply regard translation activities as linguistic conversion behaviors. Instead, we must deeply tap the political, cultural and literary points of the region in which the translated language is located, The translation is the translator's interpretation of the above multivariate results, we can say that the translation directly reflects the concentrated translation of the political, economic and cultural characteristics. 
In this case, the key issue of translation is to change from the traditional "how do we translate?" To "what is the translation doing?" In this case, simply relying on the role of experience is far from enough. Therefore, to summarize the experience is crucial. Although practice comes from experience, the obvious defects in experience can not be ignored. Experience is often easily caught in the vicious circle of low-level exploration and reasoning, which makes it difficult to have universal significance in translation practice Guidance and guidance.

Generally speaking, the specific translation experience does not directly rise to translation theory. Only when it rises to the theoretical level of abstraction can the translation theory system come into being and guide the translation practice. To sum up, the translation theory seems to be independent and separate from the translation practice. The theory is abstract. It is not as intuitive as the translation practice, and it is difficult to provide a simple and direct answer to the problems encountered in the translation practice.

In the process of actual research, the discussion of different levels of translation shows a kind of division. In the case of the same extension, there are still some correlations in different sets of independent categories: (1) awareness of whether language can be translated; (2) Method of understanding. Through relevant research and analysis, there is only an indirect correlation between the translation theory at the level of epistemology and the concrete transcoding taking into account the parameters.

In the process of carrying out daily work, we should proceed from reality and adopt corresponding theories to guide people's practical activities. In the process of translation work, translation theory is general and translation practice is a concrete activity. Therefore, in the process of translation, translators should actively analyze an article and strengthen the use of the principles in translation theory. Meanwhile, , But also need to consider all aspects of content, the only way to ensure that the translation theory to translators play a guiding role. For the time being, some people still have the illusory mentality, believing that the West is advanced and copying the corresponding translation theories, resulting in failure to achieve good results. Therefore, in the process of the application of translation theory, we can not deviate from the traditional culture of our country, we should actively learn the essence of its existence and combine it with our own traditional culture to form a theoretical system of translation theory that suits the actual situation in our country. To enhance the applicability of translation theory can we ensure the integration of translation theory and translation practice so that translation theory can better guide the translation practice and further promote the translation work. In addition, we must also ensure the unity of translation theory and translation practice so as to prevent the two from becoming disconnected.

\section{Conclusion}

To sum up, the constant development of society has brought about more frequent economic and cultural exchanges among various countries. Translation is the main bridge for exchanges between countries. Each country's languages are able to transcode each other, the information contained in each language is also different, technical translation theory to consider the special parameters of language and translation of the specific process, the only way to better and actively promote The quality of language translation work. In addition, in the process of carrying out the research on translation theory, researchers should also actively strengthen the combination of theory and practice, apply the relevant contents of translation theory to practical translation work, and timely improve the problems exposed to ensure that The applicability of translation theory meets the translation needs of various languages and realizes the rapid development of language translation.

\section{References}

[1] Sun Guiying. Intertextual Translation in the Perspective of "Relevance Translation Theory" [J]. Shandong Foreign Language Teaching. 2006 (01)

[2] Song ZP. Translation: Choice and Adaptation - Pragmatic Adaptation Theory from the 
perspective of translation studies [J]. China Translation. 2004 (02)

[3] Zhang Xinhong, HE natural. Pragmatic translation: pragmatics theory in translation [J]. Modern Foreign Languages. 2001 (03)

[4] He Ran, Ran Yongping. Relevance Theory - The Basis of Cognitive Pragmatics [J]. Modern Foreign Languages 1998 (03)

[5] He Ran, Ran Yongping. Relevance Theory - The Basis of Cognitive Pragmatics [J]. Modern Foreign Languages 1998 (03) 